\title{
High strain rate tensile and compressive effects in glassy polymers
}

\author{
J.L. Jordan ${ }^{1}$, C.R. Siviour ${ }^{2}$, and B.T. Woodworth ${ }^{1}$ \\ ${ }^{1}$ Air Force Research Laboratory, Eglin AFB, FL 32542, USA \\ ${ }^{2}$ Department of Engineering Science, University of Oxford, Parks Road, Oxford, UK
}

\begin{abstract}
Polymers are increasingly used in impact and complex high rate loading applications. Generally, the mechanical response of glassy polymers under high strain rates has been determined in compression. Some research programs have studied the combined effects of temperature and strain rate, still primarily in compression, providing better understanding of the physics behind the observed response and enhancing the models for these materials. However, limited data are available in tension, and even more limited are data describing both the compressive and tensile response of the same glassy polymer. This paper investigates the compressive and tensile response of glassy polymers across a range of stain rates from quasi-static to dynamic. Experimental results from dynamic mechanical analysis, quasi-static compression and tension, and split Hopkinson tension/pressure bars on several representative glassy polymers will be presented. The pressure dependant yield in these materials will be discussed through comparison of the tensile and compressive yield stresses.
\end{abstract}

\section{Introduction}

Since the early 1990s, a range of experimental data have been generated describing the response of glassy polymers to high strain rate loading in compression. More recently, research programs that study the combined effects of temperature and strain rate have made significant steps in providing better understanding of the physics behind the observed response [1,2], and also in modeling this response $[3,4]$. However, limited data are available in tension, and even more limited are data describing both the compressive and tensile response of the same polymer [5-8]. In studies that examine tensile response, there are, often, large gaps in the strain rate dependence. These gaps are due to the relative difficulty of performing characterization experiments in tension, especially on polymers and especially at high rates of strain. Tension testing of brittle polymers is even more challenging due to the low strains to failure, which can result in invalid tests due to failure outside the gauge length and susceptibility to bending. For example, although experimental data exists on epoxy in compression across a range of strain rates [2,9]; very little data exists in tension $[6,10]$. In order to achieve valid tension tests on epoxy at high strain rates, pulse shaping techniques have been developed $[6,10]$. Additionally, digital image correlation coupled with high speed photography has been used to measure the strain state of the entire sample in situ [10].

One important reason for addressing the gap in strainrate dependant tensile data is that polymers exhibit pressure dependant yield, which has been measured in the past using complex loading apparatus $[11,12]$. However, comparison of the tensile and compressive yield stresses of individual polymers can also result in the determination of the hydrostatic pressure dependence in these materials $[3,6,13]$.

\section{Experimental approach}

Four glassy polymers were investigated in this study impact resistant (Type II) polyvinyl chloride (PVC), and polycarbonate (PC), which were in the form of $25.4 \mathrm{~mm}$ diameter extruded rod that was machined into specimens of the appropriate dimensions. Right circular cylinders were used for all compression experiments, with the quasi-static experiments using $8 \mathrm{~mm} \times 8 \mathrm{~mm}$ samples and the medium rate and dynamic experiments using $8 \mathrm{~mm}$ diameter by $3.5 \mathrm{~mm}$ samples. The samples for tensile experiments were based on the design by Gerlach, et al. [6] and were designed with a shortened gauge length and reduced radius of curvature in order to promote sample failure within the gauge length.

Dynamic Mechanical Analysis (DMA) samples (60 mm long $\times 12.5 \mathrm{~mm}$ wide $\times 3.2 \mathrm{~mm}$ thick) were tested in dual cantilever configuration in a TA Instruments Q800 at frequencies of 1,10 , and $100 \mathrm{~Hz}$, displacements of 5 , 10,15 , and $25 \mu \mathrm{m}$ and a temperature range of $-100{ }^{\circ} \mathrm{C}$ to $190{ }^{\circ} \mathrm{C}$.

The quasi-static compression and tension experiments were conducted using a screw-driven Houndsfield load frame at strain rates from $10^{-3} \mathrm{~s}^{-1}$ to $10^{-1} \mathrm{~s}^{-1}$. The strain was measured using a laser extensometer (Fiedler Optoelectronik Model P-50), which has a resolution of $0.1 \mu \mathrm{m}$. For the compression experiments, stripes were tracked on the compression anvils; for the tensile experiments, stripes were painted on the samples themselves.

A custom-built hydraulic load frame was used to access strain rates $1-50 \mathrm{~s}^{-1}$ in both tension and compression. A Linear Variable Differential Transformer (LVDT) was used to measure the displacement of the sample. Additionally, a DRS Lightening DigiStreak camera was used to image the tensile experiments. As in the quasi-static experiments, stripes were painted on the specimen, which were recorded by the camera and used to calculate strain in the gauge length.

High strain rate compression experiments were performed using a Split Hopkinson Pressure Bar (SHPB); similarly, high strain rate tension experiments were performed using a Split Hopkinson Tension Bar (SHTB). In both configurations, a gas driven projectile is used to impact either the end of the input bar in compression or a flange mounted on the end of the input bar in tension.

This is an Open Access article distributed under the terms of the Creative Commons Attribution License 2.0, which permits unrestricted use, distribution, and reproduction in any medium, provided the original work is properly cited. 

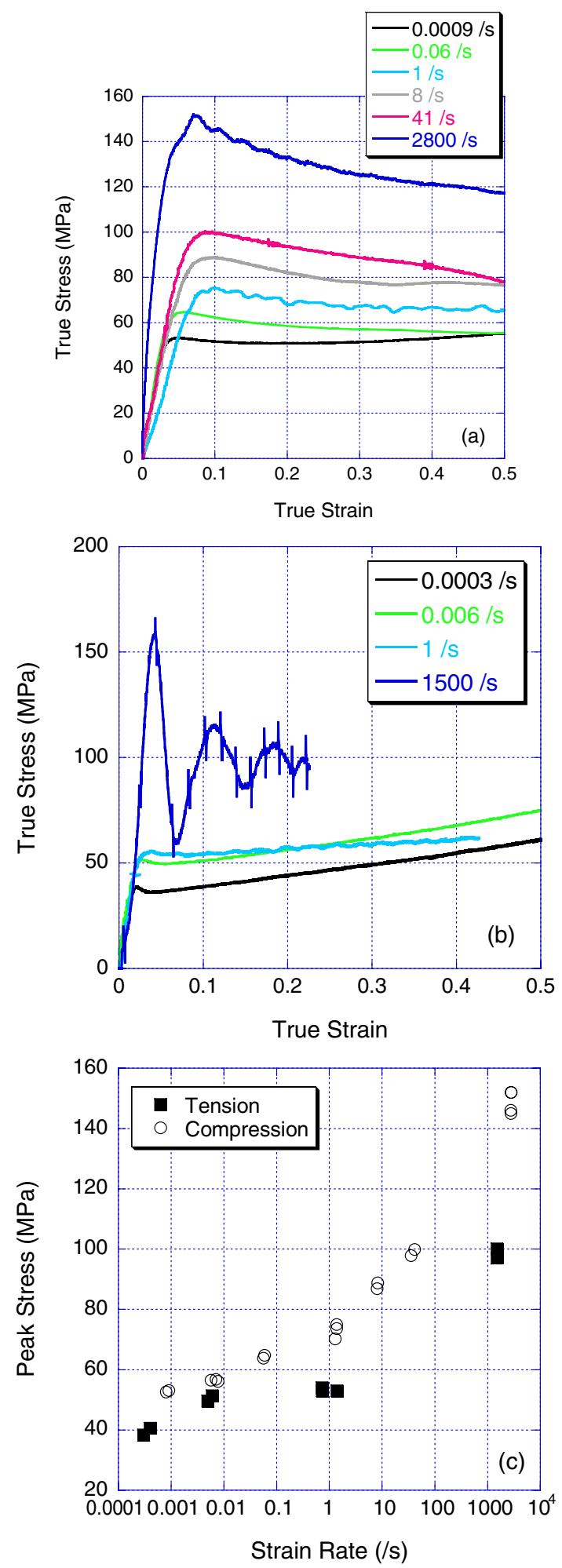

Fig. 1. (a) Compressive stress-strain response and (b) tensile stress-strain response of PVC with (c) peak stress versus strain rate.

For the SHPB system, the input and output bars are made of 6061-T6 aluminum. In the SHTB system, the input bar is Ti6Al4V and the output bar is phosphor bronze. A stress wave (compressive or tensile) then travels through the input bar until it reaches the sample. At the sample, part of the wave is reflected and part is transmitted. A pulse shaper, in the form of a preloading bar, is used on the SHTB system in order to increase the rise time of the input wave and therefore smooth the wave, in particular removing a stress peak, which often occurs [8].

The stress waves are measured using strain gauges mounted on the bars. The SHPB system uses two sets of strain gauges mounted mid-way along the length of the input and output bars. The data are acquired and analyzed as described in [14]. It is valuable to ensure that the sample is in equilibrium [15]; this was done in all experiments. The SHTP system uses three sets of strain gauges in order to record the stress waves - two on the input bar and one on the output bar. The use of three gauges allows for longer input pulses while using shorter input and output bars. The gauge signals are used to calculate the force at and velocity of the two specimen-bar interfaces using and analysis in [16]. These are, in turn, used to derive the stress and strain in the specimen. Additionally, photographs were taken of the deforming sample in order to provide a further measure of strain. A Specialised Imaging SIMX16 high speed camera was used to take 16 pictures through the duration of the loading, and an edge detection algorithm was then used to track the position of stripes painted on the specimen surface.

\section{Results and discussion}

\subsection{Polyvinyl chloride (PVC)}

The compressive and tensile response of the PVC used for this study has been reported previously [14]; the relevant results are discussed here for comparison with the other glassy polymers. Figure 1(a) and (b) shows the compressive and tensile stress-strain response of PVC. The behavior shown is typical for a glassy polymer - an initial elastic region, followed by a non-linear elastic region and yield, then strain softening followed by strain hardening. At higher strain rates, the strain softening is masked by thermal softening due to the transition between isothermal and adiabatic test conditions [4].

The compressive and tensile peak stress as a function of strain rate is plotted in figure 1(c). The relationship between strain rate and peak stress appears to be linear over the range of strain rates tested. Using simple extrapolation, the dynamic mechanical analysis of PVC indicated that a $\beta$ phase transition, due to the restrictions in secondary change motions [3], would not be observed at room temperature until strain rates approaching 7000/s [14]. However, the large increase in stress for both SHPB and SHTB experiments indicate that the $\beta$ phase transition is playing a role at these strain rates. This is consistent with the phase transition in similar polymers [2,3].

\subsection{Polycarbonate (PC)}

The dynamic mechanical analysis of PC, figure 2 , showed that the glass transition $(\alpha)$ varies from $152^{\circ} \mathrm{C}$ at $1 \mathrm{~Hz}$ to $163^{\circ} \mathrm{C}$ at $100 \mathrm{~Hz}$. The lower temperature $\beta$ phase transition shifts from $-92^{\circ} \mathrm{C}$ to $-71^{\circ} \mathrm{C}$ as the frequency varies from $1 \mathrm{~Hz}$ to $100 \mathrm{~Hz}$. This data agrees very well with that of 


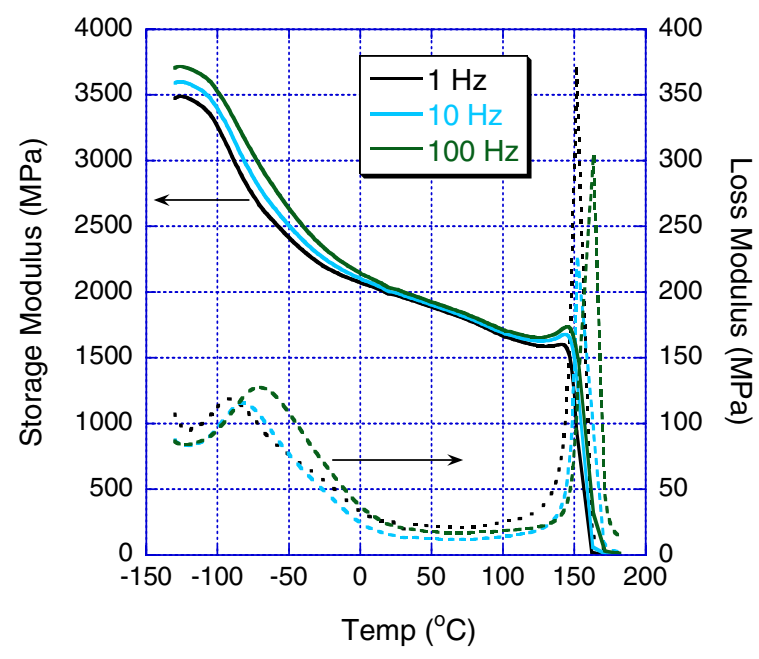

Fig. 2. Dynamic mechanical analysis of PC.

Mulliken and Boyce [3]. The $\beta$ phase transition increases more rapidly than the glass transition over the same frequency range due to the lower activation energy for the $\beta$ phase transition. The testing frequency, displacement, and gauge length can be used to determine the equivalent strain rate for the DMA results [3]. Using the decompose-shiftreconstruct method described by Mulliken and Boyce [3], extrapolation of the $\beta$ phase transition to room temperature indicates that the phase transition would be evident in compression and tension testing at $\sim 100 / \mathrm{s}$. The compressive and tensile response of $\mathrm{PC}$ as a function of strain rate is shown in figure 3(a) and (b), respectively. For both loading regimes, the stress increases with strain rate, as expected. As with PVC, the behavior shown is typical for a glassy polymer - an initial elastic region, followed by a non-linear elastic region and yield, then strain softening followed by strain hardening.

There are oscillations in the high strain rate tensile experiments, which are believed to be an experimental artifact. The "real" stress-strain response is believed to be an average line fitted through the oscillations. The difficulties inherent in performing high strain rate tensile experiments are evident in this and the PVC data sets, and work is ongoing to improve the quality of data obtained.

Figure 3(c) presents the peak stress at yield for both loading regimes. For many glassy polymers, including PC, the $\beta$ phase transition results in an increased yield strength under high strain rate loading [2,3]. The extrapolation from DMA indicates that this transition is evident at $\sim 100$ /s, which is difficult to confirm with the data presented in Figure 3(c), since there is a large gap in the available test data around this strain rate. However, the large jump in peak stress for the SHPB and SHTP results indicate that the $\beta$ phase transition may be having an effect.

\section{Conclusions}

Polyvinyl chloride (PVC) and polycarbonate (PC) have been experimentally studied in tension and compression varying strain rate. Dynamic mechanical analysis indicates the presence of two phase transitions in both polymers.
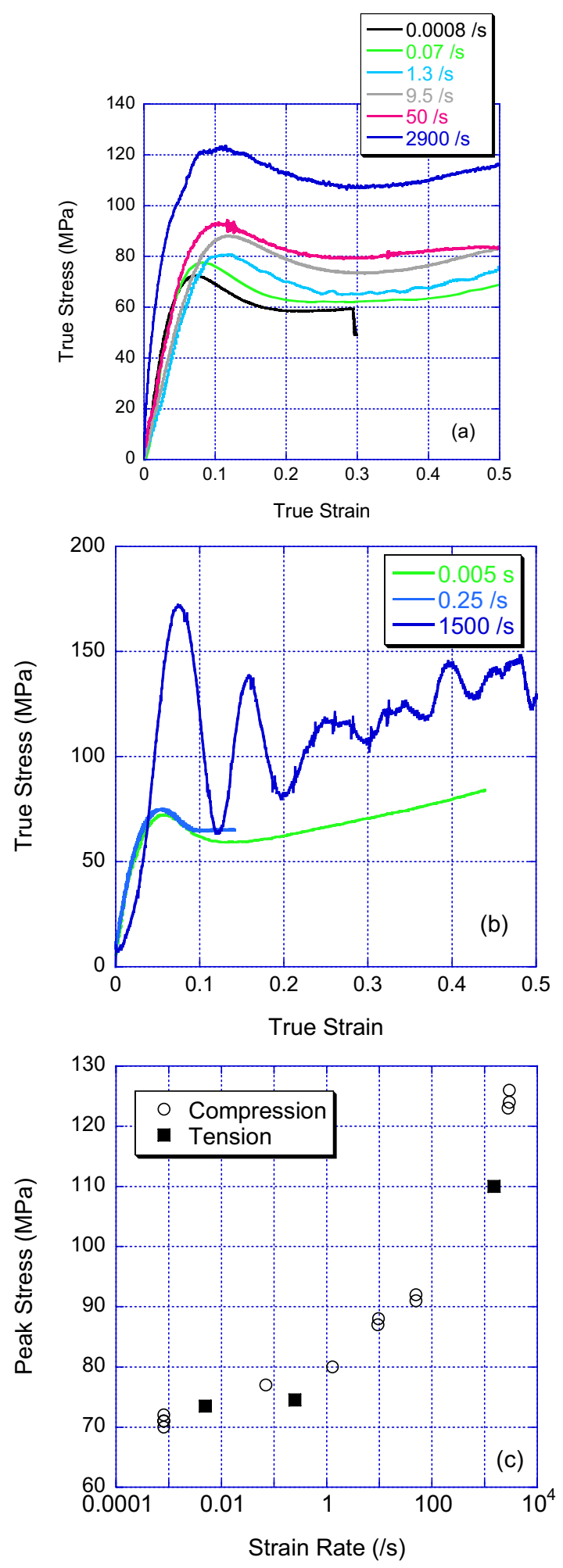

Fig. 3. (a) Compressive stress-strain response and (b) tensile stress-strain response of PC with (c) peak stress versus strain rate.

The compressive and tensile stress-strain response across a range of strain rates for both PVC and PC are typical for glassy polymers. The compressive yield stress is consistently higher than the tensile yield stress. For both polymers, a bilinear behavior as a function of strain rate is indicated. However, additional testing and analysis is required to confirm these results. 


\section{Acknowledgements}

The authors would like to thank the Air Force Office of Scientific Research for support.

\section{References}

1. J.L. Jordan, J.R. Foley, and C.R. Siviour, Mechanics of Time Dependant Materials, 12, 249 (2008).

2. C.R. Siviour, C.R., S.M. Walley, W.G. Proud, and J.E. Field, Polymer, 46, 12546 (2005).

3. A.D. Mulliken and M.C. Boyce, International Journal of Solids and Structures, 43, 1331 (2006).

4. A.D. Mulliken, S.Y. Soong, M.C. Boyce, and R.E. Cohen, J. Phys. IV France, 134, 217 (2006).

5. W. Chen, F. Lu, and M. Cheng, Polymer Testing, 21, 113 (2002).
6. R. Gerlach, C.R. Siviour, N. Petrinic, and J. Wiegand, Polymer, 49, 2728 (2008).

7. C.M. Roland, J.N. Twigg, Y. Vu, and P.H. Mott, Polymer, 48, 574 (2007).

8. S. Sarva, A.D. Mulliken, and M.C. Boyce, J. Phys. IV France, 134, 95 (2006).

9. H. Lu, G. Tan, and W. Chen, Mechanics of Time Dependant Materials," 5, 119 (2001).

10. W. Chen, F. Lu, and M. Cheng, Polymer Testing, 21, 113 (2002).

11. D.R. Mears, K.D. Pae, and J.A. Sauer, Journal of Applied Physics, 40 [11], 4229 (1969).

12. J.A. Sauer, D.R. Mears, and K.D. Pae, European Polymer Journal, 6, 1015 (1970).

13. C. Bauwens-Crowet, J-C. Bauwens, and G. Homes, Journal of Materials Science, 7, 176 (1972).

14. J.L. Jordan, C.R. Siviour, and B.T. Woodworth, Shock Compression of Condensed Matter - 2011, (to be published). 